\title{
Atrophic Pap Smears, Differential Diagnosis and Pitfalls: A Review
}

\author{
Ali Dastranj Tabrizi ${ }^{*}$
}

\begin{abstract}
As a unique preventable cancer, invasive cervical carcinoma is the second most common cancer among the women in developing countries. Change in women's lifestyle, worldwide spread of HPV infection and women's lack of effective education and screening programs in less developed countries constitute the most important factors for its high incidence and mortality rate. From a diagnostic perspective, atrophic smears may be interpreted as positive malignant smears in postmenopausal and occasionally in premenopausal women. However, rarely a high grade or malignant smear might be rendered as the atrophic smear. For this reason acquaintance with atrophic smears and their diagnostic challenges should be included in pathology and cytotechnology training courses. In this review, usual pattern of atrophic smears and differential diagnosis of atrophic smears along with mimics will be presented for decision making and particularly avoiding overdiagnosis.
\end{abstract}

Keywords: Atrophic Pap smear, Invasive cervical cancer, HPV infection

\section{Introduction}

Although cervical cancer incidence has been dramatically declined in developed countries due to the implementation of systematic and organized screening into health care systems, this preventable malignancy comprises the second most common cancer in women in developing countries (1). Change in women's life style, world wide spread of HPV (human papilloma virus) infection and women's lack of effective education and screening systems in less developed countries constitute the most important factors for the very high incidence and mortality rate in less developed societies (2-4). The most common subtypes of high risk HPVs involving in cervical carcinogenesis are HPV 16 and HPV 18 although other subtypes of high risk HPVs may be detected as dominant carcinogenic agent. On the other hand, low sensitivity and specificity of Pap smear particularly in conventional type impose serious consequences including false negative and false positive results (5).

From a diagnostic perspective, atrophic smears may be misinterpreted as positive smears in postmenopausal and occasionally in perimenopausal women. However, rarely a high grade or malignant smear might be rendered as an atrophic smear. Fortunately, introduction of new techniques in the field of Pap smear including new collection devices and automated machines leads to better results. This review will discuss general cytologic features of atrophic smears and a number of common morphologic mimics of atrophic smears which can lead to overinterpretation. The structure of this review is as follows: (a) a brief inspection of usual pattern of atrophic smears, $(b)$ main differential diagnosis which can mimic atrophic smears, $(c)$ pitfalls which may lead to over interpretation, and finally, $(d)$ suggestive strategies for correct decision making

\section{Usual Pattern of Atrophic Smears}

Taking Pap smear in postmenopausal women occasionally shows a hypocellular background and may display the absence of endocervical or transformation zone components (6,7) (Figure 1A). Microscopic examination of atrophic smears which is typically seen in postmenopausal women usually shows numerous parabasal and intermediate cells that may be arranged in small clusters or as individual cells (Figure 1B and C). A small number of superficial cells may be seen. Presence of a large number of superficial cells along with intermediate cells may indicate the need for hormone replacement therapy or uterine prolapse treatment. In these circumstances, parakeratotic cells may also be seen and should not be considered as evidence of low grade squamous intraepithelial lesion (LSIL). The parabasal cells usually show a relatively small amount of cytoplasm and high nuclear/cytoplasmic ratio. The condensed nuclear chromatin leads to nuclear hyperchromasia, however, no chromatin clumping and no nucleoli is seen. Absence of chromatin clumping, sharp and regular nuclear membrane and absence of mitotic figure are hallmarks of atrophic smears which allow easy distinction of these cases from high grade intraepithelial or malignant lesions (Figure 
$1 \mathrm{D}, 1 \mathrm{E})$. Clusters of endocervical cells usually are seen in well-taken atrophic smears although their absence should not be rendered as an insufficient specimen. Usually, the normal atrophic smears show some debris hanging from the cytoplasmic border. These materials should not be considered as the evidence of malignancy which is seen widely in squamous cell carcinoma. The absence of polymorphonuclear (PMN) debris, clean background of smears, close adherence of these materials to cytoplasmic membrane and absence of nuclear atypia are helpful criteria for distinction between atrophic and malignant smears (Figure 1F).

\section{Differential Diagnosis}

A. Invasive Squamous Cell Carcinoma

Although misdiagnosis of an atrophic smear as a malignant smear is uncommon even in inexpert hands, extreme caution should be used to avoid this misinterpretation. Sheaths of parabasal cells with high nuclear/cytoplasmic (N/C) ratio and hyperchromatic nuclei may simulate malignant cells $(8,9)$. Information about patients' clinical history, pelvic examination and colposcopic findings along with careful attention to the appearance of nuclear chromatin and nuclear outlines will be useful (Figure $1 \mathrm{G})$. Monotonous appearance of cellular sheaths, uniform nuclear chromatin, absence of mitotic figures, lack of tumor diathesis and nonexistence of tad pole cells all are in favor of benign atrophic smear versus malignant lesion. In contrast, in a malignant smear from an invasive squamous cell carcinoma, the malignant cells dispersed haphazardly in the background of some normal appearing cells. Although the dirty background of the smear is one of the most distinguishing features of malignant smears due to tumor cell necrosis, some granular material derived from degenerating parabasal cells are usually present in the background of atrophic smears $(10,11)$. Therefore close attention should be paid to find marked nuclear pleomorphism, coarse nuclear chromatin, ragged nuclear membranes and relatively easily found mitotic figures for the establishment of the diagnosis of invasive squamous cell carcinoma.

\section{B. High Grade Squamous Intraepithelial Lesion}

Atrophic smears are prone to be misinterpreted as HSIL. In HSIL the smear background is clean. Reactive parabasal cells, especially in atrophic vaginitis, may show some nuclear membrane irregularity and more hyperchromasia. Awareness of patient's clinical history and familiarity with reactive changes in an atrophic smear such as nucleomegaly, the presence of cytoplasmic vacuoles and cytoplasmic polychromasia usually lead to correct diagnosis. Monotonous appearance of smear, evenly distributed chromatin, and smooth nuclear membrane in the majority of cells are the main clues to make a correct diagnosis (Figure 1D, 1F). In doubtful cases, appreciation of colposcopic appearance of the cervix, patient's clinical

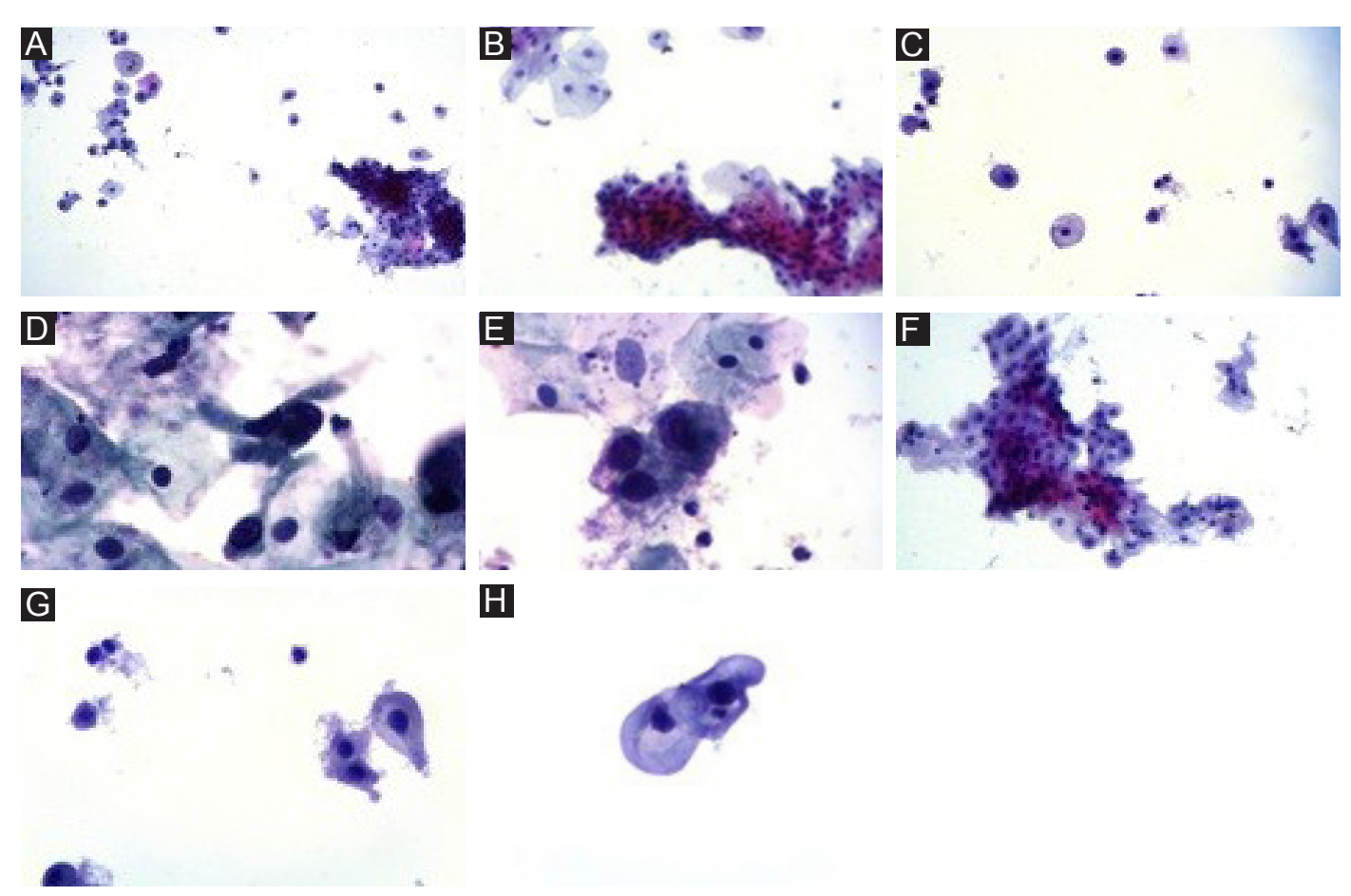

Figure 1. An atrophic smear showing hypocellular background (A). Sheaths of parabasal and intermediate cells that arranged in small clusters (B) or in individual cells pattern (C). Large nuclear/cytoplasmic ratio, clumped nuclear chromatin in high grade squamous intraepithelial lesion (D). Coarse chromatin clumping in a high grade lesion (E). Absence of chromatin clumping, sharp and regular nuclear membrane, absence of nuclear debris and mitotic activity are hallmarks of atrophic smears (F). Typical parabasal cells in an atrophic smear $(G)$. Typical koilocytes in low grade squamous intraepithelial lesions $(H)$. 
setting and HPV typing usually solve the problem. In some cases, consultation with a clinician and trying a short course of vaginal estrogen along with repeated smear will be needed (12).

\section{Low Grade Squamous Intraepithelial Lesion}

Occasionally some of the atrophic smears may contain epithelial cells with vacuolated cytoplasm and rarely perinuclear halo. Awareness of patient's age and her sexual activity, the absence of typical koilocytes with raisinoid nuclei and also the monotonous appearance of the background assist to avoid overdiagnosis (13) (Figure $1 \mathrm{H})$.

D. Atypical squamous cells of undetermined significance Misinterpretation of atrophic smears as Atypical squamous cells of undetermined significance (ASCUS) may be the most common mistake in inexperienced hands. Although most of the parabasal cells in atrophic smears show high N/C ratio, the absence of chromatin abnormality would be a helpful sign in a differential diagnosis. It should be mentioned that in the most smears with epithelial cells abnormalities, the abnormal cells show sparse distribution between normal cells. However, most of the atrophic smears show fairly monotonous appearance. It should be mentioned that ASCUS has low positive predictive value for a squamous intraepithelial lesion in perimenopausal age group and may be overused (14).

F. Reactive Changes Induced by Radiation Therapy Most of the patients with radiation change in Pap smear have atrophic smear due to postmenopausal status, previous oophorectomy and radiation-induced atrophic changes of ovaries. Therefore atrophic smears with radiationinduced atypical changes are at risk of overdiagnosis. In these cases, patient's past medical history and recognition of microscopic appearance of radiation-induced changes are valuable diagnostic criteria for ruling out of tumor recurrence.

\section{Discussion}

After menopause, decreased serum estrogen level prevents squamous epithelium maturation beyond the parabasal or lower intermediate cell and induces a thin epithelium $(15,16)$. These physiologic changes manifested in Pap smears by hypocellularity and monotonous sheaths of small parabasal cells with rounded or ovoid cytoplasm. These cells have relatively larger nuclei but overall, the parabasal cells are much smaller than intermediate cells and their cytoplasm is dense (17) (Figure 1B). In these cells, N/C ratio is relatively high, but the uniform and homogenous nuclear chromatin and smooth nuclear membrane are useful characters in differentiation from high grade squamous intraepithelial lesion or invasive squamous cell carcinoma. However, the above mentioned features are routinely used in cytology. Shidham et al (18) suggested that P16ink4 immunocytochemistry may be useful in a precise categorization of Pap smears with ASCUS or ASC-H diagnosis. In the smears taken from elderly patients with uterine prolapse, smears may contain anucleated superficial and parakeratotic cells due to cervix keratinization. For the most part, the parabasal cells are arranged in large flat sheaths which are typical of atrophy. In liquid based smears, more single parabasal cells can be seen (Figure 1C) and these cells are prone to be misinterpreted as HSIL (19). Attention to evenly distributed chromatin, smooth nuclear membrane, and absence of mitotic figure are relevant cytologic clues leading to correct diagnosis. Any nuclear abnormalities including clumped and coarse chromatin, irregular and jagged nuclear membrane, mitotic figures and apoptotic bodies should be interpreted as evidences of premalignant or malignant lesions and should be confirmed by colposcopy and biopsy. Although atrophic smears may contain anucleated cells due to uterine prolapse, the presence of atypical parakeratotic cells or cells with atypical nuclei and keratinized cytoplasm should be interpreted with extreme caution.

Although the application of the above mentioned cytologic criteria will be useful in most of these difficult cases, some investigators recommended that utilization of immunocytologic markers such as Ki67 would be effective in some of the problematic cases $(20,21)$. Other studies proposed that immunocytochemical detection of P16 and ki67 acts as a more accurate route in establishing a correct diagnosis (22-24).

A granular background which is commonly seen in atrophic smears should not be confused with the tumor diathesis which is found in the background of malignant smears due to tumor cell necrosis and ulceration (Figure 1C). Clark hypothesized that in ThinPrep slides, blocking of filter coverage by epithelial cells can be mediated by this debris and inflammatory cells which leads to hypocellular smears (11).

\section{Conclusion}

Although in most cases the diagnosis of atrophic smears is straightforward, in rare cases particularly in inexperienced hands it may be the source of misinterpretation such as ASCUS, HSIL or even squamous cell carcinoma. On the other hand, some cases of cervical squamous cell carcinoma may be diagnosed as atrophic smears especially in rapid screening. Therefore, considering patient's clinical findings along with the colposcopic appearance of the cervix would be useful in problematic cases. Moreover, use of proliferative and oncologic markers such as Ki67 and P16 could be valuable in making a correct diagnosis.

\section{Conflict of Interests}

Author declares that he has no conflict of interests. 


\section{Ethical Issues}

Not applicable.

\section{Financial Support}

None to be declared.

\section{References}

1. Torre LA, Bray F, Siegel RL, Ferlay J, Lortet-Tieulent J, Jemal A. Global cancer statistics, 2012. CA Cancer J Clin. 2015;65(2):87-108. doi:10.3322/caac.21262

2. Vaccarella S, Lortet-Tieulent J, Plummer M, Franceschi $\mathrm{S}$, Bray F. Worldwide trends in cervical cancer incidence: impact of screening against changes in disease risk factors. Eur J Cancer. 2013;49(15):3262-3273. doi:10.1016/j. ejca.2013.04.024

3. Bruni L, Diaz M, Castellsague X, Ferrer E, Bosch FX, de Sanjose S. Cervical human papillomavirus prevalence in 5 continents: meta-analysis of 1 million women with normal cytological findings. J Infect Dis. 2010;202(12):1789-1799. doi:10.1086/657321

4. Forman D, de Martel C, Lacey CJ, et al. Global burden of human papillomavirus and related diseases. Vaccine. 2012;30 Suppl 5:F12-23. doi:10.1016/j.vaccine.2012.07.055

5. Chen C, Yang Z, Li Z, Li L. Accuracy of several cervical screening strategies for early detection of cervical cancer: a meta-analysis. Int J Gynecol Cancer. 2012;22(6):908-921. doi:10.1097/IGC.0b013e318256e5e4

6. Davey DD, Cox JT, Austin RM, et al. Cervical cytology specimen adequacy: patient management guidelines and optimizing specimen collection. J Low Genit Tract Dis. 2008;12(2):71-81. doi:10.1097/LGT.0b013e3181585b9b

7. Waddell CA. The influence of the cervix on smear quality. I: Atrophy. An audit of cervical smears taken post-colposcopic management of intraepithelial neoplasia. Cytopathology. 1997;8(4):274-281.

8. Selvaggi SM. Atrophic vaginitis versus invasive squamous cell carcinoma on ThinPrep cytology: can the background be reliably distinguished? Diagn Cytopathol. 2002;27(6):362364. doi:10.1002/dc.10204

9. Bulten J, de Wilde PC, Boonstra H, Gemmink JH, Hanselaar AG. Proliferation in "atypical" atrophic pap smears. Gynecol Oncol. 2000;79(2):225-229. doi:10.1006/gyno.2000.5943

10. Ziabkowski TA, Naylor B. Cyanophilic bodies in cervicovaginal smears. Acta Cytol. 1976;20(4):340-342.

11. Clark SB, Dawson AE. Invasive squamous-cell carcinoma in ThinPrep specimens: diagnostic clues in the cellular pattern. Diagn Cytopathol. 2002;26(1):1-4.

12. Bateson DJ, Weisberg E. An open-label randomized trial to determine the most effective regimen of vaginal estrogen to reduce the prevalence of atrophic changes reported in postmenopausal cervical smears. Menopause.
2009;16(4):765-769. doi:10.1097/gme.0b013e318193e7cd

13. Jovanovic AS, McLachlin CM, Shen L, Welch WR, Crum CP. Postmenopausal squamous atypia: a spectrum including "pseudo-koilocytosis". Mod Pathol. 1995;8(4):408-412.

14. Keating JT, Wang HH. Significance of a diagnosis of atypical squamous cells of undetermined significance for Papanicolaou smears in perimenopausal and postmenopausal women. Cancer. 2001;93(2):100-105.

15. Repse-Fokter A, Takac I, Fokter SK. Postmenopausal vaginal atrophy correlates with decreased estradiol and body mass index and does not depend on the time since menopause. Gynecol Endocrinol. 2008;24(7):399-404. doi:10.1080/09513590802217290

16. Robboy SJ, Anderson MC, Morse A. Cervical precancer (intraepithelial neoplasia). In: Robboy SJ, Anderson MC, Russell P, eds. Pathology of the female reproductive tract. London: Churchill Livingstone; 2002:165-93.

17. Reichert RA. Diagnostic Gynecologic and Obstetric Pathology: An Atlas and Text. Philadelphia: Lippincott Williams Wilkins; 2012.

18. Shidham VB, Mehrotra R, Varsegi G, D’Amore KL, Hunt B, Narayan R. p16 immunocytochemistry on cell blocks as an adjunct to cervical cytology: Potential reflex testing on specially prepared cell blocks from residual liquid-based cytology specimens. Cytojournal. 2011;8:1. doi:10.4103/1742-6413.76379

19. Kalof AN, Cooper K. Our approach to squamous intraepithelial lesions of the uterine cervix. J Clin Pathol. 2007;60(5):449-455. doi:10.1136/jcp.2005.036426

20. Ejersbo D, Jensen HA, Holund B. Efficacy of Ki-67 antigen staining in Papanicolaou (Pap) smears in postmenopausal women with atypia--an audit. Cytopathology. 1999;10(6):369-374.

21. Fakhrjou A, Dastranj-Tabrizi A, Ghojazadeh $\mathrm{M}$, et al. Diagnostic value of protein Ki67 (MIB-1) in atypical pap smears of postmenopausal women. Asian Pac J Cancer Prev. 2013;14(8):4815-4818.

22. Rokita W, Skawinski D, Zmelonek-Znamirowska A, et al. [Results of pap smears and immunocytochemical detection of the p16 and Ki67 proteins in women with cervical intraepithelial neoplasia and cervical cancer]. Ginekol Pol. 2012;83(11):822-826.

23. Wentzensen N, Fetterman B, Tokugawa D, et al. Interobserver reproducibility and accuracy of p16/Ki-67 dual-stain cytology in cervical cancer screening. Cancer Cytopathol. 2014;122(12):914-920. doi:10.1002/cncy.21473

24. Trutnovsky G, Kolovetsiou-Kreiner V, Reich O. p16/Ki67 dual-stained cytology testing may predict postpartum outcome in patients with abnormal papanicolaou cytology during pregnancy. Acta Cytol. 2014;58(3):293-296. doi:10.1159/000358817

Copyright $\odot 2018$ The Author (s); This is an open-access article distributed under the terms of the Creative Commons Attribution License (http://creativecommons.org/licenses/by/4.0), which permits unrestricted use, distribution, and reproduction in any medium, provided the original work is properly cited. 